\title{
VARIACIONES EN ÁREA FOLIAR Y CONCENTRACIONES DE CLOROFILAS Y NUTRIMENTOS ESENCIALES EN HOJAS DE CAFÉ ROBUSTA (Coffea canephora P.) DURANTE UN CICLO ANUAL
}

\section{VARIATIONS IN THE FOLIAR AREA AND CONCENTRATIONS OF CHLOROPHYLLS AND ESSENTIAL NUTRIENTS IN LEAVES OF ROBUSTA COFEE (Coffea canephora P.) DURING AN ANNUAL CYCLE}

\author{
Marín-Garza, T. ${ }^{1}$; Gómez-Merino, F.C. ${ }^{2}$; Aguilar-Rivera, N. ${ }^{1}$; Murguía-González, J. ${ }^{1}$; \\ Trejo-Téllez, L.I. ${ }^{3} ;$ Pastelín-Solano, M.C. ${ }^{4}$; Castañeda-Castro, O. $^{4^{*}}$
}

\begin{abstract}
${ }^{1}$ Facultad de Ciencias Biológicas y Agropecuarias. Camino Peñuela-Amatlán de los Reyes km 1, Peñuela, Amatlán de los Reyes, Veracruz. C. P. 94945. ${ }^{2}$ Colegio de Postgraduados Campus Córdoba. Carretera Federal Córdoba-Veracruz km 348, Manuel León, Amatlán de los Reyes, Veracruz. C. P. $94946 .{ }^{3}$ Colegio de Postgraduados Campus Montecillo. Carretera México-Texcoco km. 36.5 Montecillo, Texcoco, Estado de México. C. P. 56230. ${ }^{4}$ Universidad Veracruzana. Facultad de Ciencias Químicas. Prolongación de Oriente 6 No. 1009. Orizaba, Veracruz. C. P. 94340.
\end{abstract}

*Autor de correspondencia: odcastaneda@uv.mx

\section{RESUMEN}

Las hojas son el principal órgano donde se lleva a cabo la fotosíntesis en la planta y su composición química puede variar de acuerdo a sus etapas de desarrollo. En esta investigación se midió el área foliar, la concentración de clorofila y de nutrimentos en hojas de café Robusta (Coffea canephora) de las variedades Robmex y FRT-07 en diferentes etapas del cultivo: vegetativa, floración y fructificación. El área foliar $\left(\sim 140 \mathrm{~cm}^{2}\right)$ fue mayor en la variedad Robmex durante la etapa de floración. En esta misma variedad se tuvieron mas medias más altas de clorofila a (2.00 $\mathrm{mg} \mathrm{g}^{-1} \mathrm{PMF}$ ) y total (2.83 mg $\mathrm{g}^{-1} \mathrm{PMF}$ ), así como de $\mathrm{Cu}\left(47.0 \mathrm{mg} \mathrm{kg}^{-1}\right.$ ) en la etapa vegetativa. Con excepción del Cu, todos los demás nutrimentos esenciales fueron más altos en la variedad FRT-07.

Palabras clave: Café, variedad Robusta, área foliar, clorofila, nutrimentos esenciales.

Agroproductividad: Vol. 11, Núm, 4, abril. 2018.pp: 36-41. Recibido: noviembre, 2017. Aceptado: abril, 2018 


\begin{abstract}
Leaves are the main organ where photosynthesis takes place in the plant, and their chemical composition can vary according to their stages of development. In this study, the foliar area was measured, as well as the concentration of chlorophyll and nutrients in Robusta coffee (Coffea canephora) leaves of the varieties Robmex and FRT-07 in different stages of growth: vegetative, flowering and fructification. The leaf area $\left(140 \mathrm{~cm}^{2}\right)$ was larger in the Robmex variety during the flowering stage. In this same variety, there were higher means of chlorophyll a (2.00 $\mathrm{mg} \mathrm{g}^{-1}$ FBW) and total (2.83 $\mathrm{mg} \mathrm{g}^{-1} \mathrm{FBW}$ ), and of $\mathrm{Cu}\left(47.0 \mathrm{mg} \mathrm{kg}^{-1}\right.$ ) in the vegetative stage. With the exception of $\mathrm{Cu}$, all other essential nutrients were higher in the FRT-07 variety.
\end{abstract}

Keywords: Coffee, Robusta variety, foliar area, chlorophyll, essential nutrients.

\section{INTRODUCCIÓN}

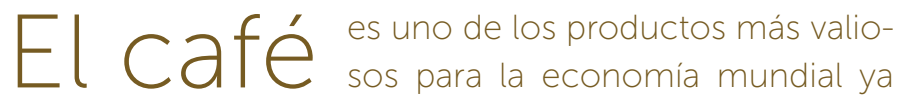
que genera empleos a millones de personas, y para muchos países representa hasta el 80 \% de sus ingresos por exportación (ICO, 2017). El cultivo de café en México es tan importante que está relacionado con el aspecto económico, social y ambiental, dando sustento a más tres millones de connacionales que están vinculados de alguna forma con el café, generando divisas, en promedio de 900 millones de dólares anuales (SAGARPA, 2015). De los 3.8 millones de sacos (de $60 \mathrm{~kg}$ ) de café que se producen en México, el 96 \% es café Arabica y el restante $4 \%$ es café Robusta, con lo que el país se ubica dentro de los 10 principales productores y exportadores de este grano y es el íder en producción de café orgánico (FIRA, 2015; USDA, 2017).

La planta de café de la variedad Robusta es un arbusto que puede alcanzar 10 metros de altura, tiene una raíz poco profunda y con granos pequeños, en comparación con los de la especie Arabica, además su sabor es más fuerte y amargo, ya que duplica el contenido de cafeína (hasta $4.5 \%$ ), aunque se considera de calidad inferior. Algunas variedades de Robusta son valoradas por su sabor profundo y su alta calidad (FIRA, 2015).
Una de las ventajas del café Robusta (Coffea canephora), es que su producción es mayor (hasta 4 t ha $^{-1}$ de grano al año), presenta tolerancia a plagas y cambios climáticos, se requieren menor cantidad de café cereza para obtener un quintal de café oro (González-Concepción y Rubio-Pérez, 2014), además de la importancia que tiene en la industria del café soluble, por la mayor cantidad de sólidos solubles que lo hace más productivo que otras especies (Ribeiro et al., 2014).

De acuerdo con Hernández-Falcón et al. (2015) el café no solo se consume por su sabor, sino también porque tiene muchos beneficios para la salud humana incluyendo la reducción de riesgos a enfermedades como cirrosis hepática, diabetes tipo dos, cáncer colorrectal y hepático, así como enfermedades cardiovasculares, y neurodegenerativas (i.e. Parkinson y Alzheimer) (Fonseca-García et al., 2014).

La composición química del grano depende de la actividad fotosintética en la hoja, y los contenidos de biomoléculas como carbohidratos, lípidos, proteinas y clorofilas de la hoja dependen de varios factores incluyendo la etapa fenológica. De hecho, durante la fructificación, nutrimentos como $\mathrm{N}, \mathrm{K}$ y Mg se movilizan de las hojas a los frutos (Sadeghian et al., 2012), lo que indica que las concentraciones de éstos en tejido foliar también fluctuan a través del ciclo de desarollo de la planta (Sadeghian y Salamanca, 2015)

En este estudio se cuantificaron las fluctuaciones del área foliar, concentración de clorofilas y macro y micro nutrimentos en hojas de plantas de café Robusta (Coffea canephora) de las variedades Robmex y FRT-07 en un ciclo anual.

\section{METODOLOGÍA}

\section{Material vegetal y condiciones para el experimento}

El presente trabajo se realizó con hojas de plantas de café de las variedades Robmex y FRT-07 de la especie Coffea canephora. Las plantas de café tenían cinco años de edad, provenientes de parcelas demostrativas de la finca La Laja, ubicada en la congregación La Laja, municipio de Tlaltetela, Veracruz, México, a 19¹7' 10.8" de latitud norte, y $96^{\circ}$ 56' 51.9" de longitud oeste, y 1130 m.s.n.m. Las plantas se cultivan bajo sol, con el método tradicional selectivo (siembra, fertilización y poda), suelo tipo cambisol con plantaciones de 2000 arbustos por hectárea. Las etapas del ciclo anual del cultivo evaluadas 
fueron: vegetativa (febrero), floración (mayo) y fructificación (diciembre).

\section{Medición del área foliar}

El área foliar se midió con el instrumento óptico medidor de área foliar CID-Biocience modelo Cl-202 (Camas, WA, EEUU) que determina indirectamente el índice de área foliar (IAF).

\section{Análisis de clorofilas}

Se midió la concentración de clorofilas (clorofila a, b y total) en hojas de café siguiendo la metodología descrita por Harborne (1973). Tejido foliar fresco se colocó en acetona al 80 \%, se mantuvo en maceración por 24 horas a $4{ }^{\circ} \mathrm{C}$, se filtró y se cuantificó el contenido de clorofilas con base al peso de biomasa fresca (PBF) a una absorbancia de 645 y 665 nm en el espectrofotómetro ThermoFisher modelo Genesys 10S UV-VIS (Madison, WI, EEUU).

\section{Análisis nutrimental}

La concentración de N en hoja se realizó por el método micro-Kjeldahl (Bremner, 1965) y los nutrimentos P, K, Ca, Mg, Fe, $\mathrm{Cu}, \mathrm{Zn}, \mathrm{Mn}$, Fe y B se cuantificaron por espectrofotometría de emisión óptica de inducción por plasma acoplado, en un equipo ICP-OES modelo 725 (Agilent; Mulgrave, Australia), siguiendo la metodología descrita por Alcántar y Sandoval (1999).

\section{Diseño experimental y análisis estadístico}

El experimento tuvo un diseño factorial $2 \times 3$, en donde el factor 1 fue la variedad a dos niveles (Robmex y FRT-07) y el factor 2 fue la etapa fenológica (vegetativa, floración, fructificación). Los tratamientos se distribuyeron completamente al azar, y con los datos obtenidos se realizó un análisis de varianza y prueba de comparación de medias de Tukey $(P \leq 0.05)$ con el paquete estadístico SAS (SAS, 2011).

\section{RESULTADOS Y DISCUSIÓN}

La mayor área foliar se presentó en la variedad Robmex durante la etapa de floración. Los demás tratamientos observaron medias estadísticamente similares entre ellas, indepenientemente de la variedad y de la etapa de evaluación, aunque numéricamente, la menor área se observó en la variedad FRT-07 durante la floración (Figura 1).

De acuerdo con Montoya-Restrepo et al. (2017) el área foliar se relaciona con la tasa fotosintética, evapotranspiración, y desarrollo vegetativo, así como con la absorción de agua y nutrimentos.

Estudios realizados entre etapas fisiologías de distintos cultivos, han observado que los valores más altos del área foliar se dan poco después del inicio de floración o durante esta etapa (Acosta-Díaz et al., 2008; RodríguezCabello et al., 2015). De acuerdo con Arcila et al. (2007), esto se debe a que una parte de las funciones de las hojas es la de proteger a las yemas, flores y frutos de la radiación y el medio ambiente adverso. El aumento del área foliar en el árbol, se relaciona con la fructificación y la producción (Almenares-Garlobo et al., 2015). De acuerdo con Montoya-Restrepo et al. (2017), la relación del área foliar con la producción de café cereza indica que por cada $100 \mathrm{~cm}^{2}$ de área foliar existe un incremento de $2.37 \mathrm{~g}$ de café cereza verde aproximadamente.

Los tratamientos también afectaron las concentra-

ciones de clorofilas (Cuadro 1). La mayor concentración de clorofila a (2.0 $\mathrm{mg} \mathrm{g}^{-1}$ PMF) y total (2.8 $\mathrm{mg} \mathrm{g}^{-1} \mathrm{PMF}$ ) se observó en la variedad Robmex en etapa vegetativa. En todos los casos, la menor concentración de las tres variables (clorofila a, b y total) se observó en la variedad FRT-07 durante la etapa de floración.

En cítricos, Reyes-Santamaría et al. (2000) observaron que la mayor tasa fotosintética se lleva a cabo durante los meses de octubre a febrero, en donde la planta acumuló carbohidratos como fuente de reserva y para utilizarlos durante el crecimiento vegetativo, floración y fructificación. De acuerdo a Gonçalves de Oliveira et al. (2009), un óptimo contenido de clorofila en hojas de café es necesaria para el adecuado proceso de fotosíntesis. 


\begin{tabular}{|c|c|c|c|c|}
\hline \multirow{2}{*}{ Variedad } & \multirow{2}{*}{ Etapa } & Clorofila a & Clorofila b & Clorofila total \\
\hline & & \multicolumn{3}{|c|}{$\mathrm{mg} \mathrm{g}^{-1} \mathrm{PMF}$} \\
\hline \multirow{3}{*}{ Robmex } & Vegetativa & $2.00 \pm 0.53 a$ & $0.80 \pm 0.35 b$ & $2.83 \pm 0.87 a$ \\
\hline & Floración & $1.76 \pm 0.89 c$ & $1.00 \pm 0.39 a$ & $2.57 \pm 0.86 c$ \\
\hline & Fructificación & $1.45 \pm 0.98 d$ & $0.56 \pm 0.67 d$ & $2.03 \pm 0.87 d$ \\
\hline \multirow{3}{*}{ FRT-07 } & Vegetativa & $1.89 \pm 0.64 b$ & $0.74 \pm 0.86 c$ & $2.66 \pm 0.69 b$ \\
\hline & Floración & $0.83 \pm 0.47 f$ & $0.33 \pm 0.67 f$ & $1.17 \pm 0.74 f$ \\
\hline & Fructificación & $1.24 \pm 0.37 \mathrm{e}$ & $0.48 \pm 0.87 e$ & $1.74 \pm 0.86 \mathrm{e}$ \\
\hline
\end{tabular}

Medias \pm DE con letra distinta en cada columna indican diferencias estadísticas significativas $(P \leq 0.05)$ por etapa del ciclo de cultivo. PMF: Peso de la Materia Fresca.

Los factores de estudio y sus niveles también afectaron la concentración de los macronutrimentos evaluados en hojas (Cuadro 2). En ambas variedades, las mayores concentraciones de $\mathrm{N}$ se observaron durante la etapa de fructificación. Para el caso de $\mathrm{P}$, hubo un efecto marcado de la variedad, más que de la etapa, con la variedad FRT-07 mostrando los mayores niveles de este nutrimento en hoja. El K observó una mayor fluctuación entra tratamientos, con los mayores niveles en la variedad FRT-07 durante la fase vegetativa, y los menores en Robmex durante la etapa vegetativa. Ca y Mg mostraron un comportamiento muy semejante entre ellos, pues las mayores cocentraciones se observaron en la variedad FRT-07 durante la etapa de floración, en tanto que los niveles más bajos se regitraron en Robmex durante la fructificación.

Se sabe que los requerimientos de nutrientes y agua de las plantas cambian de acuerdo a su etapa de desarrollo durante del año (Ramírez et al., 2010). Por ser requerido para la división y elongación celular, el $\mathrm{N}$ es mayormente movilizado durante las etapas tempranas del crecimiento y desarrollo (Monsalve et al., 2009). Dubberstein et al. (2016) observaron que en hojas de plantas de café al iniciar la fructificación los niveles de $\mathrm{N}$ y $\mathrm{P}$ fueron más altos, mientras que las concentraciones de $\mathrm{Ca}, \mathrm{Mg}$ y $\mathrm{S}$ se observaron variaciones a través del tiempo y que estaban más relacionados con los nutrimentos disponibles en el suelo y el clima, así como de la especie y variedad de la planta que con el desarrollo en etapa de fructificación (Sadeghian et al., 2012).

En plantas de café, los nutrimentos guardan una estrecha relación de la hoja a la fruta, con un año de alta producción y otro de bajo rendimiento debido al agotamiento de fotoasimilados y nutrimentos, particularmente el N, P y K (Reis et al., 2009).

En cuanto al contenido de los micronutrimentos, durante la etapa vegetativa se observó la mayor concentración de Fe (283.8 $\mathrm{mg} \mathrm{kg}^{-1}$ ) en la variedad FRT-07, así como $\mathrm{Cu}\left(47.87 \mathrm{mg} \mathrm{kg}^{-1}\right.$ ) en la variedad Robmex en la etapa vegetativa. El Zn (22.3 $\left.\mathrm{mg} \mathrm{kg}^{-1}\right)$ destacó en la variedad

\begin{tabular}{|c|c|c|c|c|c|c|}
\hline \multirow{2}{*}{ Variedad } & \multirow{2}{*}{ Etapa } & $N$ & P & K & $\mathrm{Ca}$ & $\mathrm{Mg}$ \\
\hline & & \multicolumn{5}{|c|}{$\mathrm{g} \mathrm{kg}^{-1} \mathrm{PMS}$} \\
\hline \multirow{3}{*}{ Robmex } & Vegetativa & $21.10 \pm 0.94 c$ & $0.78 \pm 0.69 b$ & $9.37 \pm 0.85 \mathrm{e}$ & $10.65 \pm 0.87 d$ & $2.36 \pm 0.55 \mathrm{~cd}$ \\
\hline & Floración & $23.80 \pm 0.94 d$ & $0.99 \pm 0.65 b$ & $13.20 \pm 0.59 d$ & $13.01 \pm 0.54 \mathrm{c}$ & $2.52 \pm 0.69 c$ \\
\hline & Fructificación & $23.90 \pm 0.90 b$ & $0.99 \pm 0.90 b$ & $14.93 \pm 0.65 b$ & $10.63 \pm 0.86 d$ & $2.14 \pm 0.97 d$ \\
\hline \multirow[t]{3}{*}{ FRT-07 } & Vegetativa & $20.50 \pm 0.96 \mathrm{e}$ & $1.05 \pm 0.90 \mathrm{ab}$ & $17.86 \pm 0.58 a$ & $12.99 \pm 0.69 c$ & $3.32 \pm 0.86 b$ \\
\hline & Floración & $20.80 \pm 0.95 d$ & $1.06 \pm 0.68 \mathrm{a}$ & $9.50 \pm 0.54 \mathrm{e}$ & $20.35 \pm 0.69 a$ & $4.22 \pm 0.96 a$ \\
\hline & Fructificación & $26.00 \pm 0.86 a$ & $1.22 \pm 0.54 a$ & $13.70 \pm 0.86 c$ & $17.88 \pm 0.65 b$ & $3.20 \pm 0.97 b$ \\
\hline
\end{tabular}

Medias \pm DE con letra distinta en cada columna indican diferencias estadísticas significativas ( $P \leq 0.05)$ por etapa del ciclo de cultivo. PMS: Peso de la Materia Seca. 
FRT-07 durante la floración, así como el Mn 1471.6 mg $\mathrm{kg}^{-1}$ ) y $\mathrm{B}\left(62.5 \mathrm{mg} \mathrm{kg}^{-1}\right)$, en la misma variedad en la etapa de fructificación (Cuadro 3).

Los micronutrimentos son elementos con funciones fisiológicas esenciales en el metabolismo de las plantas. Algunos de ellos funcionan como cofactores enzimáticos o como activadores en la formación estructural de la planta (Dechen y Nachtigall, 2006). De la misma forma a lo observado en macronutrimentos, las mayores concentraciones de micronutrimentos se registraron principalmente en la variedad FRT-07. Estas fluctuaciones pueden ser atribuídas a cambios en el medio ambiente, la disponibilidad hídrica, la especie y variedad de la planta (Sadeghian y Salamanca, 2015).

La disminución en la concentración foliar de micronutrimentos es debido a que el requerimiento de éstos por la planta es mayor a su tasa de absorción, lo que produce una dilución de nutrimentos en algunos órganos (Meza y Pire, 2008). Sin embargo, las tendencias registradas en los análisis foliares en café, no siempre son concluyentes, lo que puede ser atribuible al hecho de que los nutrimentos requeridos por los frutos, los proporcionan principalmente las hojas cercanas a los frutos, las cuales no corresponden a las que se muestrean para su análisis químico que regularmente son el tercer o cuarto par de hojas de la rama (Sadeghian y Salamanca, 2015).

Es importante destacar que las concentraciones tanto de macronutrimentos como de micronutrimentos observados en este estudio están dentro del rango reportado por Dubberstein et al. (2016).

\section{CONCLUSIONES}

anto el factor variedad como la etapa del cultivo
influyen en las variables evaluadas. Durante la eta-
pa vegetativa las hojas de café en la variedad Robmex presentaron las concentraciones más altas de clorofila a y total, y Cu, mientras que en la etapa de floración se observó la mayor área foliar. Las concentraciones más altas de N y P se observaron en FRT-07 durante la fructificación. El K fue más abundante en FRT durante la fase vegetativa. Ca y Mg fueron mayores en FRT durante la floración. Los micronuttrimentos mostraron un comportamiento similiar a los macronutrimentos, dado que a excepción del Cu, todos los demás mostraron concentraciones más elevadas en la variedad FRT-07.

\section{LITERATURA CITADA}

Acosta-Díaz E., Acosta-Gallegos J.A., Ramírez A., Domingo M., PadillaRamírez J.S. 2008. Relación entre índice de área foliar y rendimiento en frijol bajo condiciones de secano. Agr. Téc. Méx. 34: 13-20

Alcántar G., Sandoval V. 1999. Manual de análisis químico de tejido vegetal. Publicación Especial Núm. 10. Sociedad Mexicana de la Ciencia del Suelo. Chapingo. Estado de México. 156 p.

Almenares-Garlobo G.R., Pérez-Hernández M.C., Torres-de la Noval W. Varela-Nualles M., Pavón-Rosales M.I. 2015. Characterization of the vegetative development and its relationship with fruiting and production of orange trees [Citrus sinensis (L.) Osbeck]. Cult. Trop. 36: 2 56-61.

Arcila J., Farfán F., Moreno A., Salazar L.F., Hincapié E. 2007. Sistemas de producción de café en Colombia. Cenicafé 7: 144-160

Bremner J. 1965. Total nitrogen. Agronomy 9: 1149-1178

Dechen A.R., Nachtigall G.R. 2006. Micronutrientes. In: Fernandes, M. S. Nutrição mineral de plantas. Sociedade Brasileira de Ciência do Solo. Viçosa, Minas Gerais, Brasil. pp. 327-354.

Dubberstein D., Partelli F.L., Dias J.R.M., Espindola, M.C. 2016 Concentration and accumulation of macronutrients in leaf of coffee berries in the Amazon, Brazil. Austr. J. Crop Sci. 10: 701710

\begin{tabular}{|c|c|c|c|c|c|c|}
\hline \multirow{2}{*}{ Variedad } & \multirow{2}{*}{ Etapa } & $\mathrm{Fe}$ & $\mathrm{Cu}$ & $\mathrm{Zn}$ & $\mathrm{Mn}$ & B \\
\hline & & \multicolumn{5}{|c|}{$\mathrm{mg} \mathrm{kg}^{-1} \mathrm{PMS}$} \\
\hline \multirow{3}{*}{ Robmex } & Vegetativa & $170.09 \pm 0.60 c$ & $47.87 \pm 0.50 \mathrm{a}$ & $5.89 \pm 0.78 f$ & $251.77 \pm 0.65 d$ & $32.18 \pm 0.76 f$ \\
\hline & Floración & $124.30 \pm 0.95 d$ & $7.90 \pm 0.95 \mathrm{e}$ & $8.71 \pm 0.65 \mathrm{e}$ & $243.64 \pm 0.53 \mathrm{e}$ & $36.05 \pm 0.94 \mathrm{e}$ \\
\hline & Fructificación & $111.43 \pm 0.96 f$ & $8.32 \pm 0.86 d$ & $10.32 \pm 0.68 c$ & $158.00 \pm 0.69 f$ & $45.21 \pm 0.43 d$ \\
\hline \multirow{3}{*}{ FRT-07 } & Vegetativa & $283.85 \pm 0.79 a$ & $18.75 \pm 0.95 b$ & $19.41 \pm 0.94 b$ & $296.25 \pm 0.76 c$ & $47.72 \pm 0.78 c$ \\
\hline & Floración & $120.62 \pm 0.65 \mathrm{e}$ & $7.50 \pm 0.86 f$ & $22.32 \pm 0.96 a$ & $312.84 \pm 0.68 b$ & $54.11 \pm 0.85 b$ \\
\hline & Fructificación & $211.42 \pm 0.69 b$ & $14.25 \pm 0.69 c$ & $9.49 \pm 0.45 d$ & $471.63 \pm 0.63 a$ & $62.58 \pm 0.76 a$ \\
\hline
\end{tabular}

Medias \pm DE con letra distinta en cada columna indican diferencias estadísticas significativas $(P \leq 0.05)$ por etapa del ciclo de cultivo. PMS: Peso de la Materia Seca. 
FIRA. 2015. Panorama Agroalimentario 2015. Café. Fideicomisos Instituidos en Relación con la Agricultura. https://www. gob.mx/cms/uploads/attachment/file/61949/Panorama_ Agroalimentario_Caf_2015.pdf

Fonseca-García L., Calderón-Jaimes L.S., Rivera M.E. 2014. Capacidad antioxidante y contenido de fenoles totales en café y subproductos del café producido y comercializado en norte de santander (Colombia). Vitae 21: 228-236.

Gonçalves de Oliveira J., da Costa Aguiar Alves P.L., Pierre Vitória A. 2009. Alterations in chlorophyll a fluorescence, pigment concentrations and lipid peroxidation to chilling temperature in coffee seedlings. Environ. Exper. Bot. 67: 71-76.

González-Concepción O., Rubio-Pérez A. 2014. Cambio climático y la percepción de los campesinos sobre la variedad café Robusta En la zona de "La Torcaza", Sierra Maestra, Cuba. Batey: Rev. Cub. Antropol. Sociocult. 6: 110-119

Harborne J.B. 1973. Phytochemical methods. London: Chapman and Hall.

Hernández-Falcón D., Ledea-Lozano O.E., Fernández-García L.A., González-García E. 2015. Validación de un método cromatográfico para la determinación de cafeína en muestras acuosas de la Industria Farmacéutica. Rev. Cub. Farmac. 49 219-231

ICO. 2017. International Coffee Organization. Statistics. http://www ico.org/trade_statistics.asp? section=Statistics

Meza N., Pire, R. 2008. Evaluación de la nutrición mineral del níspero (Manilkara achras Miller Fosberg) en plantas jóvenes cultivadas en contenedores. Rev. Fac. Agron. 25: 496-506.

Monsalve J., Escobar R., Acevedo M., Sánchez M., Coopman R. 2009 Efecto de la concentración de nitrógeno sobre atributos morfológicos, potencial de crecimiento radical y estatus nutricional en plantas de Eucalyptus globulus producidas a raíz cubierta. Bosque 30: 88-94

Montoya-Restrepo E., Hernández-Arrefondo J., Unigarro-Muñoz C., Flórez-Ramos C. 2017. Estimación del área foliar en café variedad Castillo a libre exposición y su relación con la producción. Cenicafé 68: 55-61.

Ramírez V.H., Arcila J., Jaramillo A., Rendón J.R., Cuesta G., Menza H. Sánchez P. 2010. Floración del café en Colombia y su relación con la disponibilidad hídrica térmica y de brillo solar. Cenicafé 61: $132-158$

Reis A. R., Favarin J.L., Gallo L.A., Malavolta E., Morales M.F., Lavres J. 2009. Nitrate reductase and glutamine synthetase activity in coffee leaves during fruit development. Rev. Brasil. Cien. Solo 33: 315-324

Reyes-Santamaría M.I., Villegas-Monter M.A., Colinas-León M.T. Calderón-Zavala G. 2000. Peso específico, contenido de proteína y de clorofila en hojas de naranjo y tangerino. Agrociencia 34: 49-55.

Ribeiro B., Mendonça L., Assis G., Mendonça J., Malta M., Montanari F. 2014. Evaluation of the chemical and sensory characteristics of Coffea canephora Pierre and Coffea arabica L. blends. Coffee Sci. 9: 178-186

Rodríguez-Cabello J., Díaz-Hernández Y., Pérez-González A., Fundora L.R., Rodríguez-Hernández P. 2015. Análisis del crecimiento de un genotipo silvestre de Carica papaya L. cultivado ex situ y cv. "Maradol Roja". Cult. Trop. 36: 96-105

Rodríguez-Larramendi L.A., Guevara-Hernández F., Gómez-Castro H., Fonseca-Flores M., Gómez-Castañeda J.C., Pinto-Ruiz R. 2016 Leaf anatomy related to photosynthetic pathway in coffee trees (Coffea arabica L., var. Caturra Rojo) exposed to different solar radiation levels at Sierra Maestra, Granma, Cuba. Acta Agron. 65: 248-254

Sadeghian K.S., Mejia M.B., González O.H. 2012. Acumulación de nitrógeno, fósforo y potasio en los frutos de café. Cenicafé 63: 07-18.

Sadeghian K.S. 2010. Fertilización: Una práctica que determina la producción de los cafetales. Avan. Téc. Cenicafé 391: 1-8.

Sadeghian S., Salamanca A. 2015. Micronutrimentos en frutos y hojas de café. Avances Cenicafé 66: 73-87

SAGARPA. 2015. Secretaría de Agricultura, Ganadería, Desarrollo Rural, Pesca y Alimentation. https://www.gob.mx/sagarpa.

SAS. 2011. SAS/STAT® 9.3 User's Guide. SAS Institute Inc. Cary, NC, USA. 178 P.

USDA. 2017. USDA Foreign Agriculture Service. Global Agricultural Information Network. GAIN Report Number: MX7021. Mexico. Coffee Annual. Coffee Production Increase. https://gain fas.usda.gov/Recent\%20GAIN\%20Publications/Coffee\%20 Annual_Mexico\%20City_Mexico_5-25-2017.pdf

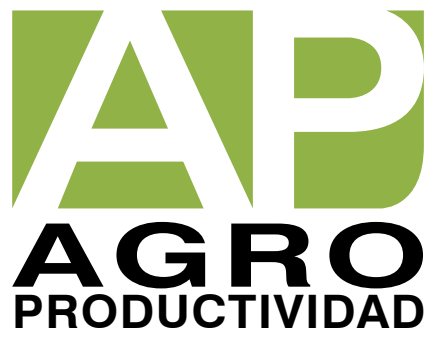

